\title{
A Novel Wideband MIMO Channel Model and Experimental Validation
}

\author{
Nelson Costa, Student Member, IEEE, and Simon Haykin, Fellow, IEEE
}

\begin{abstract}
We present a novel wideband Multiple-Input, Multiple-Output (MIMO) channel model, which we refer to as the structured model. The structured model is based on the Eigenvalue Decomposition (EVD) of the wideband channel correlation matrix. It does not assume the scatterers at the receiver fade independently of those at the transmitter. It also models correlation between delay bins in the Power Delay Profile (PDP). With preliminary data gathered using McMaster's Wideband MIMO Software Defined Radio (the WMSDR) in fixed outdoor locations, and Brigham Young University's (BYU's) wideband channel sounder in fixed indoor locations, we show good agreement between modeled and measured data. The two platforms used and the environments in which the data were collected were very different. The proposed model performed equally well with both data sets, demonstrating its robustness.
\end{abstract}

Index Terms - antenna arrays; channel capacity; channel modeling; measured channel data; multiple-input multiple-output; wideband.

\section{INTRODUCTION}

1 ultiple-Input, Multiple-Output (MIMO) systems offer the promise of increased spectral efficiency by taking advantage of multipath diversity [1], [2]. The gain of real MIMO channels over single-input, single-output (SISO) channels is highly dependent on the orientation of scatterers in the channel, and the correlations between them. We refer to this as the spatial structure of the channel. Thus, in order to better understand the gains associated with multipath diversity, we can investigate the spatial structure of the channel [3].

It has been proposed to characterize the spatial structure of the MIMO channel by modeling the correlation between channel coefficients [4]. This class of channel models is called correlative channel modeling. In the past, efforts have focused on modeling the correlations in narrowband channels. Recent literature documents an increasing move toward gathering channel data in real environments to test various narrowband channel models [5], [6], [7].

In [8], Yu et al. extend the narrowband Kronecker product model to the wideband case. The Kronecker product model assumes that the channel covariance matrix may be approximated as the Kronecker product of the transmit and

Manuscript received September 1, 2006. This work was supported in part by the Natural Science and Engineering Council (NSERC) under a Special Research Opportunity Grant, and by Defence Research and Development Canada (DRDC).

N. Costa and S. Haykin are with the Electrical and Computer Engineering Department at McMaster University, Hamilton, ON, L8S 4K1 Canada (e-mail: costa@soma.mcmaster.ca, haykin@mcmaster.ca). 
receive correlation matrices. The transmit and receive correlation matrices, which we collectively term the one-sided correlation matrices, together have fewer parameters than the channel covariance matrix. To extend the Kronecker model to the wideband case, Yu et al. assume that each delay bin in the Power Delay Profile (PDP) is independent, and apply the Kronecker model to each delay bin.

In order to gain more insight into the spatial structure of the channel, the correlation matrix can be treated using the Eigenvalue Decomposition (EVD). Weichselberger [7] develops a narrowband MIMO channel model based on this approach. He uses the eigenbases computed from the EVD of the one-sided correlation matrices as parameters in his model. He shows that his model predicts the capacity of real channels more accurately than the Kronecker model.

In the following, we present a novel wideband MIMO channel model, which is an extension of the ideas contained in [7]. We refer to this model as the structured model, as it is based on the concept of structured vector modes as presented in [9]. The structured model is unique for the following reasons. (1) The structured model is based on the EVD of the one-sided correlation matrices. (2) It considers correlation in three dimensions, namely the receivetransmit-delay space. (3) It accounts for the correlation between delay bins in the Power Delay Profile (PDP). (4) It does not assume that the scatterers at the transmitter fade independently from those at the receiver. To the best of our knowledge, this is the first wideband MIMO channel model that is based on the EVD of the channel, and is capable of modeling the correlation between delay bins. Most importantly, we verify our wideband model using real-life data from two different measurement platforms, taken in two different environments. The results show the model to be fairly accurate with respect to predicting capacity in both scenarios. It consistently outperforms the extended Kronecker model.

The rest of the paper is organized as follows. In Section II, we briefly cover relevant concepts in tensor calculus. We describe the wideband system model in Section III. In Section IV.A, we summarize both the narrowband Kronecker model, and its wideband extension. We then present Weichselberger's narrowband model in Section IV.B. To develop the structured model, we extend the concepts of Weichselberger's model to the wideband case. We present the structured model in Section V. In Section VI we discuss the MIMO Azimuth Power Spectrum (APS) and show how this tool can be used to visualize the spatial structure of the channel. Using the MIMO APS, we also give an example where the Kronecker model fails to represent the spatial structure of the channel, thus motivating our new model. In Section VII, we describe the experimental setup for two wideband platforms, McMaster's Wideband MIMO Software Defined Radio (the WMSDR) and Brigham Young University's (BYU’s) wideband channel 
sounder. We use data from both platforms to verify the structured model, and present the results in Section VIII. We conclude in Section IX.

\section{NOTATION AND RELEVANT TENSOR CALCULUS}

This section introduces notation used throughout the rest of the paper. It also introduces concepts in tensor calculus relevant to our wideband system model in Section III, and the structured model in Section V. Recasting the wideband MIMO channel gains as a tensor allows us the use of tensor decomposition [10] to derive the structured model.

\section{A. Notation}

Lower case letters with no indices attached $\{a, b, c, \ldots\}$ are scalars. Bold lower case letters $\{\mathbf{a}, \mathbf{b}, \mathbf{c}, \ldots\}$ are vectors. Bold upper case letters $\{\mathbf{A}, \mathbf{B}, \mathbf{C}, \ldots\}$ are matrices. Tensors are multidimensional generalizations of matrices. Scalars, vectors, and matrices are examples of zeroth, first, and second-order tensors. All higher order tensors are addressed using upper case calligraphic letters $\{\mathcal{A}, \mathcal{B}, \mathcal{C}, \ldots\}$. The elements of a tensor of any order can be addressed by one of two ways, e.g. the $\left(\mathrm{i}_{1}, \mathrm{i}_{2}, \mathrm{i}_{3}\right)^{\text {th }}$ element of $\mathcal{A} \in \mathbb{C}^{\mathrm{I}_{1} \times \mathrm{I}_{2} \times \mathrm{I}_{3}}$ is addressed as either $(\mathcal{A})_{\mathrm{i}_{1} \mathrm{i}_{2} \mathrm{i}_{3}}$ or $\mathrm{a}_{\mathrm{i}_{1} \mathrm{i}_{2} \mathrm{i}_{3}}$ for indices $\mathrm{i}_{1}=1, \ldots, \mathrm{I}_{1}$, $\mathrm{i}_{2}=1, \ldots, \mathrm{I}_{2}$, and $\mathrm{i}_{3}=1, \ldots, \mathrm{I}_{3}$. Throughout the paper, we use a calligraphic upper case, e.g. $\mathcal{A}$, or indexed lower case letter, e.g. $\mathrm{a}_{\mathrm{i}_{1} \mathrm{i}_{2} \mathrm{i}_{3}}$ to refer to the same tensor interchangeably. The expression $\mathcal{A} \in \mathbb{C}^{\mathrm{I}_{1} \times \ldots \times \mathrm{I}_{\mathrm{n}} \times \ldots \mathrm{I}_{\mathrm{N}}}$ implies an $\mathrm{N}^{\text {th }}$-order tensor.

\section{B. Matrix Unfolding}

There are typically many ways to map the elements of a multidimensional tensor to a matrix. One set of such mappings is termed the $\mathrm{n}^{\text {th }}$ matrix unfolding $\mathbf{A}_{(\mathrm{n})}$ of $\mathcal{A}$. Specifically, the matrix unfolding $\mathbf{A}_{(\mathrm{n})} \in \mathbb{C}^{\mathrm{I}_{\mathrm{n}} \times\left(\mathrm{I}_{n+1} \ldots \mathrm{I}_{\mathrm{N}} \mathrm{I}_{1} \ldots \mathrm{n}_{\mathrm{n}-1}\right)}$ of the tensor $\mathcal{A} \in \mathbb{C}^{\mathrm{I}_{\mathrm{t}} \times \ldots \times \mathrm{I}_{\mathrm{n}} \times \ldots \mathrm{I}_{\mathrm{N}}}$ is formed by stacking the columns formed by the $\mathrm{n}^{\text {th }}$ dimension, one after the other. The special case of a three dimensional tensor is illustrated in Fig. 1.

\section{The Vec and Unvec Operators Extended to Tensors}

The vector operator vec $(\bullet)$ can be extended to tensors. Traditionally, vec $(\mathbf{A})$ is defined as the mapping of all $a_{i j}$ to a column vector, such that the columns of $\mathbf{A}$ are stacked one on the next. The inverse operation unvec $(\mathbf{A})$ is the reverse mapping of all $\mathrm{a}_{\mathrm{ij}}$ to a matrix. In the same way, $\operatorname{vec}(\mathcal{A})$ is defined as the mapping of all $\mathrm{a}_{\mathrm{i}_{1} \ldots \mathrm{i}_{\mathrm{N}}}$ to a column vector. To illustrate this, consider a third-order tensor $\mathcal{A} \in \mathbb{C}^{\mathrm{I}_{1} \times \mathrm{I}_{2} \times \mathrm{I}_{3}}$. We first stack all the elements $\mathrm{a}_{\mathrm{i}_{1} \mathrm{i}_{3} \mathrm{i}_{3}}$ for all $\mathrm{i}_{1}$ 
while keeping $i_{2}$ and $i_{3}$ fixed. After exhausting all $i_{1}$, we increment $i_{2}$, and cycle through all $i_{1}$, with $i_{2}$ and $i_{3}$ fixed. The process is repeated until we exhaust all $i_{1}, i_{2}$, and $i_{3}$. Symbolically,

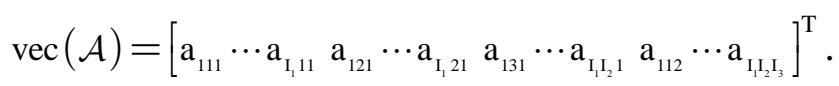

The inverse operation, unvec $(\mathcal{A})$, implies the reverse mapping.

\section{The N-mode Product}

Consider a tensor $\mathcal{A} \in \mathbb{C}^{\mathrm{I}_{1} \times \ldots \times \mathrm{I}_{n} \times \ldots \mathrm{I}_{\mathrm{N}}}$ and the matrix $\mathbf{M} \in \mathbb{C}^{\mathrm{J}_{\mathrm{n}} \times \mathrm{I}_{n}}$. The $\mathrm{n}$-mode product between them is defined as $\mathcal{B}=\mathcal{A} \times{ }_{\mathrm{n}} \mathbf{M}$, where the elements of the product tensor $\mathcal{B} \in \mathbb{C}^{\mathrm{I}_{1} \times \ldots \times \mathrm{I}_{n-1} \times \mathrm{J}_{\mathrm{n}} \times \mathrm{I}_{n+1} \times \ldots \times \mathrm{I}_{\mathrm{N}}}$ are computed as

$$
\mathrm{b}_{\mathrm{i}_{1}, \ldots \mathrm{i}_{n-1} \mathrm{j}_{\mathrm{n}} \mathrm{i}_{n+1} \ldots \mathrm{i}_{\mathrm{N}}}=\sum_{\mathrm{i}_{\mathrm{n}}} \mathrm{a}_{\mathrm{i}_{1}, \ldots \mathrm{i}_{n} \ldots \mathrm{i}_{\mathrm{N}}} \mathrm{m}_{\mathrm{j}_{\mathrm{n}_{\mathrm{n}}}} .
$$

Using the $\mathrm{n}^{\text {th }}$ unfolding of $\mathcal{A}$, the $\mathrm{n}$-mode product can also be expressed as a matrix multiplication,

$$
\mathbf{B}_{(\mathrm{n})}=\mathbf{M} \mathbf{A}_{(\mathrm{n})} \cdot
$$

\section{E. Tensor Outer Product}

The outer product of two tensors is the multiplication of all possible combinations of both tensor elements. That is, the outer product $\mathcal{A} \circ \mathcal{B}$ of two tensors $\mathcal{A} \in \mathbb{C}^{\mathrm{I}_{1} \times \ldots \times \mathrm{I}_{n} \times \ldots \mathrm{I}_{N}}$ and $\mathcal{B} \in \mathbb{C}^{\mathrm{J}_{1} \times \ldots \times \mathrm{J}_{n} \times \ldots \times \mathrm{J}_{M}}$ is $\mathcal{C} \in \mathbb{C}^{\mathrm{I}_{1} \ldots \mathrm{I}_{\mathrm{N}} \mathrm{J}_{1} \ldots \mathrm{J}_{M}}$, where

$$
\mathrm{c}_{\mathrm{i}_{1} \ldots \mathrm{i}_{\mathrm{N}} \mathrm{j}_{\ldots} \ldots \mathrm{j}_{M}}=\mathrm{a}_{\mathrm{i}_{1}, \ldots \mathrm{i}_{\mathrm{N}}} \mathrm{b}_{\mathrm{j}_{1} \ldots \mathrm{j}_{M}}
$$

for $\mathrm{i}_{\mathrm{n}}=1, \ldots, \mathrm{I}_{\mathrm{n}}$ and $\mathrm{j}_{\mathrm{m}}=1, \ldots, \mathrm{J}_{\mathrm{M}}$.

\section{F. Tensor Summation Convention}

The tensor summation convention provides a compact way of expressing a linear combination of tensor elements [11]. It states that, whenever an index appears twice in an expression, a summation over that index is implied. The summation is performed over the entire range of the repeated index.

As an example, consider the input vector $\mathbf{x} \in \mathbb{C}^{2}$, channel matrix $\mathbf{H} \in \mathbb{C}^{2 \times 2}$, and output vector $\mathbf{y} \in \mathbb{C}^{2}$. We can express the output at each $\mathrm{m}$ as the linear combination of input and channel tensor elements, i.e.

$$
\begin{gathered}
\mathrm{y}_{1}=\mathrm{h}_{11} \mathrm{x}_{1}+\mathrm{h}_{12} \mathrm{x}_{2} \\
\mathrm{y}_{2}=\mathrm{h}_{21} \mathrm{x}_{1}+\mathrm{h}_{22} \mathrm{x}_{2} .
\end{gathered}
$$

If we define $\mathrm{m}$ to be a free index, and $\mathrm{n}$ a summation index, we can rewrite the above system of equations as

$$
\mathrm{y}_{\mathrm{m}}=\sum_{\mathrm{n}=1}^{2} \mathrm{~h}_{\mathrm{mn}} \mathrm{x}_{\mathrm{n}}
$$


for $m=1,2$ and $n=1,2$. Using the summation convention, we can omit the summation symbol, and write

$$
\mathrm{y}_{\mathrm{m}}=\mathrm{h}_{\mathrm{mn}} \mathrm{x}_{\mathrm{n}} \cdot
$$

\section{WIDEBAND SYSTEM MODEL}

The SISO wideband channel is traditionally modeled as a tapped-delay line filter [12]. Consider the symbol-spaced discrete-time baseband equivalent model of Fig. 2, where $\mathrm{T}_{\mathrm{s}}$ is the sample time. The input-output relationship for a static wideband channel can be expressed as

$$
\mathrm{y}[\mathrm{i}]=\sum_{\mathrm{d}=1}^{\mathrm{D}} \mathrm{h}[\mathrm{d}] \mathrm{x}[\mathrm{i}-(\mathrm{d}-1)]+\nu[\mathrm{i}]
$$

where $\mathrm{x}[\mathrm{i}]$ is the (discrete-time) input, $\mathrm{h}[\mathrm{d}]$ is the set of channel gains for delay $d=1, \ldots, \mathrm{D}, \nu[\mathrm{i}]$ includes all noise effects, and $y[\mathrm{i}]$ is the output at instant $\mathrm{i}$. For the MIMO case, the input-output relationship can be written as

$$
\mathbf{y}[\mathrm{i}]=\sum_{\mathrm{d}=1}^{\mathrm{D}} \mathbf{H}[\mathrm{d}] \mathbf{x}[\mathrm{i}-(\mathrm{d}-1)]+\mathbf{v}[\mathrm{i}]
$$

where $\mathbf{x}[\mathrm{i}]$ is the $\mathrm{M}_{T \mathrm{x}} \times 1$ channel input for all transmitters at instant $\mathrm{i}, \mathbf{H}[\mathrm{d}]$ is the $\mathrm{M}_{\mathrm{Rx}} \times \mathrm{M}_{\mathrm{Tx}}$ channel gain matrix at delay $d, \mathbf{v}[\mathrm{i}]$ is the $\mathrm{M}_{\mathrm{Rx}} \times 1$ noise vector, and $\mathbf{y}[\mathrm{i}]$ is the $\mathrm{M}_{\mathrm{Rx}} \times 1$ receive vector.

We wish to recast the wideband MIMO model using tensors. This will allow us to use multilinear algebra to analyze the wideband channel. We note that the sequence $\mathbf{H}[\mathrm{d}]$, for all delays, can be considered as a threedimensional quantity, and is thus more easily expressed as a third-order tensor. We define the wideband H-tensor, $\mathcal{H} \in \mathbb{C}^{\mathrm{M}_{\mathrm{Rx}} \times \mathrm{M}_{\mathrm{rx}} \times \mathrm{D}}$ to be a tensor whose element $\mathrm{h}_{\text {mnd }}$ is the complex gain of the channel between receiver m, transmitter $\mathrm{n}$ at delay $d$, for $m=1 \ldots M_{R x}, n=1 \ldots M_{T x}$, and $d=1 \ldots D$. In this way, $\mathbf{H}[\mathrm{d}]$ consists of the subset of elements of $\mathcal{H}$ corresponding to delay tap d, i.e.

$$
\mathbf{H}[\mathrm{d}] \triangleq\left(\begin{array}{ccc}
\mathrm{h}_{11 \mathrm{~d}} & \cdots & \mathrm{h}_{1 \mathrm{M}_{\mathrm{Xx}} \mathrm{d}} \\
\vdots & \ddots & \vdots \\
\mathrm{h}_{\mathrm{M}_{\mathrm{Rx}} 1 \mathrm{~d}} & \cdots & \mathrm{h}_{\mathrm{M}_{\mathrm{Rx}} M_{\mathrm{Tx}} \mathrm{d}}
\end{array}\right)
$$

Note that, when $\mathrm{D}=1$, the H-tensor reduces to the familiar narrowband H-matrix $\mathbf{H} \in \mathbb{C}^{\mathrm{M}_{\mathrm{Rz}} \times \mathrm{M}_{\mathrm{Tx}}}$.

Similarly, the channel input $\mathbf{x}[\mathrm{d}]$ is a two-dimensional quantity. Thus, we can map the elements of the channel input vector $\mathbf{x}[\mathrm{d}]$ for $\mathrm{d}=0,-1, \ldots,-(\mathrm{D}-1)$ to the matrix element $\mathrm{x}_{\mathrm{nd}}$, for example, 
$\mathbf{x}[\mathrm{d}]=\left[\begin{array}{llll}\mathrm{x}_{1 \mathrm{~d}} & \mathrm{x}_{2 \mathrm{~d}} & \cdots & \mathrm{x}_{\mathrm{M}_{\mathrm{Tx}} \mathrm{d}}\end{array}\right]^{\mathrm{T}}$. Using tensor calculus, we recast (9) as

$$
\mathrm{y}_{\mathrm{m}}=\mathrm{h}_{\mathrm{mnd}} \mathrm{x}_{\mathrm{nd}}+v_{\mathrm{m}}
$$

where $\mathbf{v} \in \mathbb{C}^{\mathrm{M}_{\mathrm{nx}} \times 1}$ is the noise vector, and $\mathbf{y} \in \mathbb{C}^{\mathrm{M}_{\mathrm{R} x} \times 1}$ is the channel input. Note that we only consider the output for a single time instant.

\section{NARROWBAND CORRELATIVE MIMO CHANNEL MODELS}

Narrowband correlative models capture the spatial characteristics of a MIMO channel by modeling the correlation between each SISO channel. The full correlation matrix $\mathbf{R}_{\mathrm{H}} \in \mathbb{C}^{\mathrm{M}_{\mathrm{Rx}} \mathrm{M}_{\mathrm{Tx}} \times \mathrm{M}_{\mathrm{Rx}} \mathrm{M}_{\mathrm{Ix}}}$ is defined as

$$
\mathbf{R}_{\mathrm{H}} \triangleq \mathrm{E}\left\{\operatorname{vec}(\mathbf{H}) \operatorname{vec}^{\mathrm{H}}(\mathbf{H})\right\}
$$

In practice, $\mathbf{H}$ is computed from real channel measurements, and the expectation is approximated by the average over a finite number of H-matrices. We can generate an ensemble of synthetic Gaussian IID H-matrices using $\mathbf{R}_{\mathrm{H}}$ as

$$
\mathbf{H}_{\text {synth }}=\operatorname{unvec}\left(\mathbf{R}_{\mathrm{H}}^{1 / 2} \mathbf{g}\right)
$$

where $\mathbf{g} \in \mathbb{C}^{\mathrm{M}_{\mathrm{Rx}} \mathrm{M}_{\mathrm{x}} \times 1}$ is a vector with complex Gaussian elements, and $\mathbf{R}_{\mathrm{H}}^{1 / 2}$ is the matrix square-root of $\mathbf{R}_{\mathrm{H}}$. Because $\mathbf{H}_{\text {synth }}$ maintains the spatial correlation of $\mathbf{H}$, we say that its spatial characteristics are the same as those of the real channel. The goal of correlative channel modeling is to reduce the number of parameters needed to generate an ensemble of exemplar H-matrices, while maintaining the spatial characteristics of the measured channel.

\section{A. The Kronecker Model and Wideband Extension}

In this section, we summarize the narrowband Kronecker model, and the extension to the wideband channel, that is presented in [8]. The Kronecker model assumes that the scatterers around the receiver are not correlated with those around the transmitter. The parameters of the model are the one-sided correlation matrices $\mathbf{R}_{\mathrm{Rx}}$ and $\mathbf{R}_{\mathrm{Tx}}$. The onesided correlation matrices can be computed from the H-matrix as

$$
\mathbf{R}_{\mathrm{Rx}}=\frac{1}{\beta} \mathrm{E}\left\{\mathbf{H H}^{\mathrm{H}}\right\}, \mathbf{R}_{\mathrm{Tx}}=\frac{1}{\alpha} \mathrm{E}\left\{\mathbf{H}^{\mathrm{H}} \mathbf{H}\right\}^{\mathrm{T}},
$$

where $(\cdot)^{\mathrm{H}}$ is the Hermitian transpose, and $(\cdot)^{\mathrm{T}}$ is the ordinary transpose. In practice, the expectation is approximated by averaging over a finite number of measured channel matrices. Each correlation matrix is normalized to the average power of all channels, 


$$
\alpha \beta=\operatorname{Tr}\left(\mathbf{R}_{\mathrm{H}}\right)=\mathrm{E}\left\{\|\mathbf{H}\|_{\mathrm{F}}^{2}\right\}
$$

where $\operatorname{Tr}(\bullet)$ is the trace operator, and $\|\cdot\|_{\mathrm{F}}$ is the Frobenius norm. The Kronecker model reduces the number of parameters used to describe $\mathbf{R}_{\mathrm{H}}$ from $\left(\mathrm{M}_{\mathrm{Rx}} \mathrm{M}_{\mathrm{Tx}}\right)^{2}$ to $\mathrm{M}_{\mathrm{Rx}}^{2}+\mathrm{M}_{\mathrm{Tx}}^{2}$ by approximating it as the Kronecker product of the one-sided correlation matrices, that is,

$$
\mathbf{R}_{\mathrm{H}, \mathrm{Kron}}=\mathbf{R}_{\mathrm{Tx}} \otimes \mathbf{R}_{\mathrm{Rx}}
$$

where $\otimes$ denotes the Kronecker product. We can generate an ensemble of H-matrices by spatially filtering a complex Gaussian matrix, that is

$$
\mathbf{H}_{\text {Кгоп }}=\mathbf{R}_{\mathrm{Rx}}^{1 / 2} \mathbf{G}\left(\mathbf{R}_{\mathrm{Tx}}^{1 / 2}\right)^{\mathrm{T}}
$$

where $\mathbf{G}$ is a spatially white matrix with complex Gaussian entries. Note that, for the case when there is no correlation at the transmitter nor the receiver, $\mathbf{R}_{\mathrm{Rx}}^{1 / 2}=\mathbf{R}_{\mathrm{Tx}}^{1 / 2}=\mathbf{I}$, and the Kronecker model reduces to the Gaussian IID channel, i.e. $\mathbf{H}_{\text {Gauss }}=\mathbf{G}$.

Yu et al. [8] extend the narrowband model by applying (17) to each delay tap in the PDP by assuming the channel at each delay tap is independent of all others. The resulting model is thus

$$
\mathbf{H}_{\text {Kron }}[\mathrm{d}]=\mathbf{R}_{\mathrm{Rx}}^{1 / 2}[\mathrm{~d}] \mathbf{G}[\mathrm{d}]\left(\mathbf{R}_{\mathrm{Tx}}^{1 / 2}[\mathrm{~d}]\right)^{\mathrm{T}}
$$

where $\mathbf{R}_{\mathrm{Rx}}^{1 / 2}[\mathrm{~d}]$ and $\mathbf{R}_{\mathrm{Tx}}^{1 / 2}[\mathrm{~d}]$ are computed using the measured $\mathbf{H}[\mathrm{d}]$ for $\mathrm{d}=1, \ldots, \mathrm{D}$. In order to compare the result to the structured model, we map the elements of $\mathbf{H}_{\text {Kron }}[\mathrm{d}]$ to a third-order tensor $\mathcal{H}_{\text {Kron }} \in \mathbb{C}^{\mathrm{M}_{\mathrm{Rx}} \times \mathrm{M}_{\mathrm{Tx}} \times \mathrm{D}}$. The performance of the Kronecker model for real channels is discussed further in Section VI.B.

\section{B. The Weichselberger Model}

In this section, we provide a brief summary of the Weichselberger narrowband channel model [7]. Concepts from the Weichselberger model will be used in the derivation of the structured model.

Unlike the Kronecker model, the Weichselberger model does not assume the scatterers at the receiver are independent of those at the transmitter, making it more realistic than the Kronecker model. The parameters of the Weichselberger model are the receive and transmit eigenbases $\mathbf{U}_{\mathrm{Rx}}, \mathbf{U}_{\mathrm{Tx}}$, and the coupling matrix $\mathbf{\Omega}$. The coupling matrix describes the average energy of the SISO channel between the $\mathrm{m}^{\text {th }}$ receive and $\mathrm{n}^{\text {th }}$ transmit eigenmodes. The receive and transmit eigenbases are found by taking the EVD of the one-sided correlation matrices, 


$$
\mathbf{R}_{\mathrm{Rx}}=\mathbf{U}_{\mathrm{Rx}} \boldsymbol{\Lambda}_{\mathrm{Rx}} \mathbf{U}_{\mathrm{Rx}}^{\mathrm{H}}, \mathbf{R}_{\mathrm{Tx}}=\mathbf{U}_{\mathrm{Tx}} \boldsymbol{\Lambda}_{\mathrm{Tx}} \mathbf{U}_{\mathrm{Tx}}^{\mathrm{H}} \cdot
$$

In order to compute the coupling matrix $\boldsymbol{\Omega}$, we define an auxiliary matrix $\mathbf{K}$ as

$$
\mathbf{K}=\mathbf{U}_{\mathrm{Rx}}^{\mathrm{H}} \mathbf{H} \mathbf{U}_{\mathrm{Tx}}^{\mathrm{H}} .
$$

We can then compute the coupling matrix as

$$
\mathbf{\Omega}=\mathrm{E}\left\{\mathbf{K} \odot \mathbf{K}^{*}\right\}
$$

where $\odot$ is the Hadamard (element-wise) product, and $(\cdot)^{*}$ is the element-wise complex conjugate. The structure of $\mathbf{\Omega}$ can be directly related to the orientation of the scatterers in the channel between the transmitter and receiver [7], [9].

The above parameters lead us to the Weichselberger model synthesis equation,

$$
\mathbf{H}_{\text {Weich }}=\mathbf{U}_{\mathrm{Rx}}(\tilde{\mathbf{\Omega}} \odot \mathbf{G}) \mathbf{U}_{\mathrm{TX}}^{\mathrm{T}}
$$

where $\tilde{\boldsymbol{\Omega}}$ is the element-wise square root of $\boldsymbol{\Omega}$, and $\mathbf{G}$ is a spatially white matrix with complex Gaussian entries. Implicit in (22) is the assumption that the channel coefficients are Rayleigh distributed. The Weichselberger model reduces to the Kronecker model if and only if the coupling matrix reduces to the outer product of the transmit and receive eigenvalues, that is, if

$$
\boldsymbol{\Omega}=\frac{1}{\mathrm{P}_{\mathrm{Rx}}}\left(\begin{array}{c}
\lambda_{\mathrm{Rx}, 1} \\
\lambda_{\mathrm{Rx}, 2} \\
\vdots \\
\lambda_{\mathrm{Rx}, \mathrm{M}_{\mathrm{Rx}}}
\end{array}\right)\left(\begin{array}{c}
\lambda_{\mathrm{T} x, 1} \\
\lambda_{\mathrm{T} x, 2} \\
\vdots \\
\lambda_{\mathrm{Tx}, \mathrm{M}_{\mathrm{Tx}}}
\end{array}\right)^{\mathrm{T}}
$$

where $\lambda_{\mathrm{Rx}, \mathrm{i}}, \lambda_{\mathrm{Rx}, \mathrm{j}}$ are the eigenvalues of $\mathbf{R}_{\mathrm{Rx}}, \mathbf{R}_{\mathrm{Tx}}$, and $\mathrm{P}_{\mathrm{Rx}}=\operatorname{trace}\left(\mathbf{R}_{\mathrm{Rx}}\right)$.

Using measured data from many different scenarios, Weichselberger has shown that his model agrees closely with measured data [9]. The main disadvantages of the Weichselberger model compared to the Kronecker model are the increased number of parameters needed to synthesize an exemplar H-matrix, and the increased complexity in obtaining those parameters from the measured data. The number of parameters needed for the Weichselberger model is $\mathrm{M}_{\mathrm{Rx}} \mathrm{M}_{\mathrm{Tx}}+\mathrm{M}_{\mathrm{Rx}}^{2}-\mathrm{M}_{\mathrm{Rx}}+\mathrm{M}_{\mathrm{Tx}}^{2}-\mathrm{M}_{\mathrm{Tx}}$, whereas the number required for the Kronecker model is $\mathrm{M}_{\mathrm{Rx}}^{2}+\mathrm{M}_{\mathrm{Tx}}^{2}$. The increased complexity is the price we have to pay for a more realistic model when opting for the Weichselberger model.

\section{A NOVEL WideBAnd MIMO ChANNEL MODEL}

In this section, we present a novel wideband MIMO channel model, which we call the structured model. The 
structured model is an extension of the Weichselberger model to three dimensions. Using tensor calculus, we define tensor correlation and decomposition in three dimensions, which leads us to the structured model.

\section{A. The Wideband Correlation Matrix}

We define the sixth-order wideband correlation tensor as

$$
\mathcal{R}=\mathrm{E}\left\{\mathcal{H} \circ \mathcal{H}^{*}\right\}
$$

where the tensor outer product $\mathcal{A} \circ \mathcal{B}$ was defined in Section II.E. The elements of the wideband correlation tensor are defined as

$$
\mathrm{r}_{\mathrm{mndpqr}} \triangleq \mathrm{E}\left\{\mathrm{h}_{\mathrm{mnd}} \mathrm{h}_{\mathrm{pqr}}^{*}\right\}
$$

for $\mathrm{p}=1, \ldots, \mathrm{M}_{\mathrm{Rx}}, \mathrm{q}=1, \ldots, \mathrm{M}_{\mathrm{Tx}}$, and $\mathrm{r}=1, \ldots, \mathrm{D}$. Here we define the conjugate of all elements in $\mathcal{H}$ to be $\left(\mathcal{H}^{*}\right)_{\mathrm{mnd}} \triangleq \mathrm{h}_{\mathrm{mnd}}^{*}$

In order to make this definition more clear and to relate the result to established engineering conventions, we define the wideband correlation matrix as the expected value of the outer product of the vectorized H-tensor. The $\mathrm{M}_{\mathrm{Rx}} \mathrm{M}_{\mathrm{Tx}} \mathrm{D} \times \mathrm{M}_{\mathrm{Rx}} \mathrm{M}_{\mathrm{Tx}} \mathrm{D}$ wideband correlation matrix is computed as

$$
\mathbf{R}_{\mathrm{wB}, \mathrm{H}}=\mathrm{E}\left\{\operatorname{vec}(\mathcal{H}) \operatorname{vec}^{\mathrm{H}}(\mathcal{H})\right\}
$$

Note that $\mathbf{R}_{\text {шв, }}$ is one mapping of the elements of the wideband correlation tensor $\mathcal{R}$ to a matrix. This particular mapping has some nice properties related to real wideband channels. We can recast $\mathbf{R}_{\mathrm{WB}, \mathrm{H}}$ as

$$
\begin{aligned}
& \mathbf{R}_{\mathrm{WB}, \mathrm{H}}=\left(\begin{array}{ccc}
\frac{\mathrm{M}_{\mathrm{R}} \mathrm{M}_{\mathrm{Tx}} \times \mathrm{M}_{\mathrm{RM}} \mathrm{M}_{\mathrm{Tx}}}{\mathrm{E}\left\{\operatorname{vec}(\mathbf{H}[1]) \operatorname{vec}^{\mathrm{H}}(\mathbf{H}[1])\right\}} & \ldots & \mathrm{E}\left\{\operatorname{vec}(\mathbf{H}[1]) \operatorname{vec}^{\mathrm{H}}(\mathbf{H}[\mathrm{D}])\right\} \\
\vdots & \ddots & \vdots \\
\mathrm{E}\left\{\operatorname{vec}(\mathbf{H}[\mathrm{D}]) \operatorname{vec}^{\mathrm{H}}(\mathbf{H}[1])\right\} & \cdots & \mathrm{E}\left\{\operatorname{vec}(\mathbf{H}[\mathrm{D}]) \operatorname{vec}^{\mathrm{H}}(\mathbf{H}[\mathrm{D}])\right\}
\end{array}\right) \\
& =\left(\begin{array}{ccc}
\mathbf{R}_{\mathrm{WB}, \mathrm{H}[\mathrm{H}] \mathrm{H}[]} & \cdots & \mathbf{R}_{\mathrm{WB}, \mathrm{H}[\mathrm{l}] \mathrm{H}[\mathrm{D}]} \\
\vdots & \ddots & \vdots \\
\mathbf{R}_{\mathrm{WB}, \mathrm{H}[\mathrm{D}] \mathrm{H}[\mathrm{l}} & \cdots & \mathbf{R}_{\mathrm{WB}, \mathrm{H}[\mathrm{D}] \mathrm{H}[\mathrm{D}]}
\end{array}\right)
\end{aligned}
$$

where $\mathbf{R}_{\mathrm{WB}, \mathrm{H}[\mathrm{d}] \mathrm{H}[\mathrm{r}]} \triangleq \mathrm{E}\left\{\operatorname{vec}(\mathbf{H}[\mathrm{d}]) \operatorname{vec}^{\mathrm{H}}(\mathbf{H}[\mathrm{r}])\right\}$ for $\mathrm{r}=1, \ldots, \mathrm{D} . \mathbf{R}_{\mathrm{WB}, \mathrm{H}}$ is Hermitian symmetric. This means that the diagonal blocks of $\mathbf{R}_{\mathrm{WB}, \mathrm{H}}$ are also Hermitian symmetric, but the off-diagonal blocks are only symmetric to their counterparts across the diagonal, i.e., $\mathbf{R}_{\mathrm{WB}, \mathrm{H}[\mathrm{d}] \mathrm{H}[\mathrm{d}]}=\mathbf{R}_{\mathrm{WB}, \mathrm{H}[\mathrm{d}] \mathrm{H}[\mathrm{d}]}^{\mathrm{H}}$ and $\mathbf{R}_{\mathrm{WB}, \mathrm{H}[\mathrm{d}] \mathrm{H}[\mathrm{r}]}=\mathbf{R}_{\mathrm{WB}, \mathrm{H}[\mathrm{r}] \mathrm{H}[\mathrm{d}]}^{\mathrm{H}}$ for $\mathrm{r} \neq \mathrm{d}$.

For practical channels, $\mathbf{R}_{\mathrm{wB}, \mathrm{H}}$ tends to be close to being block diagonal, with the majority of the energy along the 
diagonal blocks $\mathbf{R}_{\mathrm{wB}, \mathrm{H}[\mathrm{d}] \mathrm{H}[\mathrm{d}]}$. To visualize why this may be so, consider the oval of scatterers model conventionally used to visualize the wideband channel, as shown in Fig. 3 [12]. Each delay tap in the PDP represents the sum of energy from all scatterers in a given annulus. For most practical channels, the scatterers in adjacent ovals are far enough away from each other that they are only weakly correlated. In this case, the cross-correlation blocks $\mathbf{R}_{\mathrm{wB}, \mathrm{H}[\mathrm{d}] \mathrm{H}[\mathrm{r}]}$ for $r \neq d$ tend to have small elements. However, as we increase the system bandwidth, the ovals get proportionally smaller as we gain spatial resolution. At some point, it can be expected that the cross correlation blocks will be significant contributors to the wideband correlation matrix. The proposed structure for $\mathbf{R}_{\mathrm{WB}, \mathrm{H}}$ covers both extremes, and allows us to visualize the correlation between scatterers at different delays.

\section{B. H-Tensor Synthesis Using the Wideband Correlation Matrix}

We define $\mathcal{H}_{\text {synth }}$ to be the synthetic H-tensor generated using $\mathbf{R}_{\mathrm{wB}, \mathrm{H}}$, i.e.

$$
\mathcal{H}_{\text {synth }}=\operatorname{unvec}\left(\mathbf{R}_{\mathrm{wB}, \mathrm{H}}^{1 / 2} \mathbf{g}\right)
$$

where $\mathbf{g} \in \mathbb{C}^{\mathrm{M}_{\mathrm{kx}} \mathrm{M}_{\mathrm{rx}} \mathrm{D} \times 1}$ is a vector whose elements are chosen independently from a complex Gaussian distribution, and $\mathbf{R}_{\mathrm{WB}, \mathrm{H}}^{1 / 2}$ is the matrix square root of $\mathbf{R}_{\mathrm{WB}, \mathrm{H}}$.

\section{One-Sided Correlation in Three Dimensions}

In order to reveal more about the structure of the channel and to develop the structured model, we extend the concept of one-sided correlation to three dimensions. We define the receive correlation matrix $\mathbf{R}_{\mathrm{Rx}} \in \mathbb{R}^{\mathrm{M}_{\mathrm{Rx}} \times \mathrm{M}_{\mathrm{Rx}}}$ to be

$$
\mathbf{R}_{\mathrm{Rx}}=\mathrm{E}\left\{\mathbf{H}_{(1)} \mathbf{H}_{(1)}^{\mathrm{H}}\right\}
$$

where $\mathbf{H}_{(1)}$ is the $1^{\text {st }}$ matrix unfolding of $\mathcal{H}$. Similarly, we define the transmit and delay correlation matrices $\mathbf{R}_{\mathrm{Tx}} \in \mathbb{R}^{\mathrm{M}_{\mathrm{Tx}} \times \mathrm{M}_{\mathrm{Tx}}}$ and $\mathbf{R}_{\mathrm{Del}} \in \mathbb{R}^{\mathrm{D} \times \mathrm{D}}$ to be

$$
\mathbf{R}_{\mathrm{Tx}}=\mathrm{E}\left\{\mathbf{H}_{(2)} \mathbf{H}_{(2)}^{\mathrm{H}}\right\}, \mathbf{R}_{\mathrm{Del}}=\mathrm{E}\left\{\mathbf{H}_{(3)} \mathbf{H}_{(3)}^{\mathrm{H}}\right\} .
$$

We can apply the EVD to each one-sided correlation matrix to obtain an eigenbasis for the receive, transmit, and delay space.

$$
\begin{aligned}
\mathbf{R}_{\mathrm{x}} & =\sum_{\mathrm{i}=1}^{\text {Limit }_{\mathrm{x}}} \lambda_{\mathrm{x}, \mathrm{i}} \mathbf{u}_{\mathrm{x}, \mathrm{i}} \mathbf{u}_{\mathrm{x}, \mathrm{i}}^{\mathrm{H}} \\
& =\mathbf{U}_{\mathrm{x}} \boldsymbol{\Lambda}_{\mathrm{x}} \mathbf{U}_{\mathrm{x}}^{\mathrm{H}}
\end{aligned}
$$


where $X \in\{R x, T x$, Del $\}$, and Limit $_{x} \in\left\{M_{R x}, M_{T x}, D\right\}$ respectively.

\section{Wideband Coupling Coefficients}

We define the wideband coupling coefficient as the average power coupled between three given eigenvectors. Thus, we define the $\mathrm{ijk} \mathrm{k}^{\text {th }}$ wideband coupling coefficient $\omega_{\mathrm{ijk}}$ as

$$
\omega_{\mathrm{ijk}}=\left(\mathbf{u}_{\mathrm{Del}, \mathrm{k}} \otimes \mathbf{u}_{\mathrm{T} x, \mathrm{j}} \otimes \mathbf{u}_{\mathrm{Rx}, \mathrm{i}}\right)^{\mathrm{H}} \mathbf{R}_{\mathrm{wB}, \mathrm{H}}\left(\mathbf{u}_{\mathrm{Del}, \mathrm{k}} \otimes \mathbf{u}_{\mathrm{Tx}, \mathrm{j}} \otimes \mathbf{u}_{\mathrm{Rx}, \mathrm{i}}\right)
$$

where $u_{R x, m i}$ is the $m^{\text {th }}$ element of the $i^{\text {th }}$ receive eigenvector, $u_{T x, n j}$ is the $n^{\text {th }}$ element of the $j^{\text {th }}$ transmit eigenvector, and $u_{\text {Del, dk }}$ is the $d^{\text {th }}$ element of the $k^{\text {th }}$ delay eigenvector, for $i=1, \ldots, M_{R x}, j=1, \ldots, M_{T x}$, and $k=1, \ldots, D$.

\section{E. The Structured Model Synthesis Equation}

Let $\mathcal{W} \in \mathbb{C}^{\mathrm{M}_{\mathrm{mx}} \times \mathrm{M}_{\mathrm{rx}} \times \mathrm{D}}$ be a tensor whose elements are computed as $\mathrm{w}_{\mathrm{mnd}}=\mathrm{g}_{\mathrm{mnd}} \sqrt{\omega_{\mathrm{mnd}}}$, where $\mathrm{g}_{\mathrm{mnd}}$ are chosen independently from a complex Gaussian distribution, and $\omega_{\mathrm{mnd}}$ is the wideband coupling coefficient. After some manipulation, we can show that the synthesis equation for the structured model is

$$
\mathcal{H}_{\text {struct }}=\mathcal{W} \times \mathbf{U}_{1} \mathbf{R x}_{2} \mathbf{U}_{\mathrm{Tx}} \times \mathbf{U}_{\mathrm{Del}} .
$$

The structured model enables us to compute an ensemble of H-tensors with approximately the same spatial characteristics as the measured channel.

\section{THE MIMO APS AND KRONECKER MODEL DEFICIENCIES}

As previously mentioned, the purpose of correlative channel models is to approximate the spatial structure of the channel. A useful tool to visualize the spatial structure is the Double Directional Azimuth Power Spectrum, which we will refer to as the MIMO APS. The MIMO APS allows us to visualize the location and magnitude of scatter clusters in the channel with respect to the receiver and transmitter azimuth. Each resolvable cluster represents a path from the transmitter to the receiver. The properties of a MIMO channel are largely determined by the spatial structure of the channel; the number and orientation of the scatterers determines the degree of spatial diversity and the multiplexing gain of the channel. Using the MIMO APS, we can qualitatively evaluate a given model's ability to approximate the spatial structure of the channel. We begin this section with a brief introduction to the MIMO APS. Using the MIMO APS, we then discuss instances where the Kronecker model does not accurately represent the spatial structure of the channel. In Section VIII.D, we also compare the Kronecker and structured model's ability to approximate the spatial structure of real channels by comparing their Azimuth Power Spectra (APSs). 


\section{A. The MIMO APS}

The MIMO APS can be computed using the Bartlett beamformer, which is defined as [9]

$$
\mathrm{P}_{\mathrm{Bat}}\left(\phi_{\mathrm{Rx}}, \phi_{\mathrm{TX}}\right)=\left[\mathbf{a}_{\mathrm{Tx}}\left(\phi_{\mathrm{Tx}}\right) \otimes \mathbf{a}_{\mathrm{Rx}}\left(\phi_{\mathrm{Rx}}\right)\right]^{\mathrm{H}} \mathbf{R}_{\mathrm{H}}\left[\mathbf{a}_{\mathrm{Tx}}\left(\phi_{\mathrm{Tx}}\right) \otimes \mathbf{a}_{\mathrm{Rx}}\left(\phi_{\mathrm{Rx}}\right)\right] .
$$

where $\mathrm{P}_{\mathrm{Batt}}\left(\phi_{\mathrm{Rx}}, \phi_{\mathrm{Tx}}\right)$ is the array factor, $\mathbf{R}_{\mathrm{H}}$ is the narrowband correlation matrix, $\phi_{\mathrm{Rx}}$ and $\phi_{\mathrm{Tx}}$ are the receive and transmit azimuth angles, and $\mathbf{a}_{\mathrm{Rx}}\left(\phi_{\mathrm{Rx}}\right)$ and $\mathbf{a}_{\mathrm{Tx}}\left(\phi_{\mathrm{Tx}}\right)$ are the $\mathrm{M}_{\mathrm{Rx}} \times 1$ receive and $\mathrm{M}_{\mathrm{Tx}} \times 1$ transmit steering vectors respectively. For the case where both the receiver and transmitter are equipped with Uniform Linear Arrays (ULAs), the steering vectors are equal to the Fourier vector

$$
\mathbf{a}_{\mathrm{x}}\left(\phi_{\mathrm{x}}\right)=\sum_{\mathrm{i}=1}^{\mathrm{M}_{\mathrm{x}}} \mathrm{e}^{-\mathrm{j}(\mathrm{i}-1) \kappa \times \cdot \sin \phi_{\mathrm{x}}}
$$

where $\mathrm{X} \in\left\{\mathrm{M}_{\mathrm{Rx}}, \mathrm{M}_{\mathrm{TX}}\right\}, \kappa=2 \pi / \lambda$ is the wave number, and $\delta$ is the antenna spacing. In this case, the Bartlett beamformer becomes the two-dimensional Fourier transform of the correlation matrix. This means that the spatial structure of the channel is entirely characterized by the correlation matrix alone.

\section{B. Kronecker Model Deficiencies}

Detailed analysis of the Kronecker model using real data has shown that it performs poorly in some situations. The accuracy of the Kronecker model decreases as we increase the number of antennas $\left(\mathrm{M}_{\mathrm{Rx}}, \mathrm{M}_{\mathrm{Tx}}>3\right)$ [9], [13]. Özcelik et al. [14] provide a detailed analysis of the joint Direction of Arrival (DoA) versus Direction of Departure (DoD) Fourier spectra from real channel measurements. It was shown that, in typical Non-Line-of-Sight (NLoS) channels, the DoD spectra are typically not independent of the DoA spectra. This implies that the scatterers at either end of the link are, in fact, correlated. The Kronecker model forces independence of the two spectra, which produces artifact paths at the vertical and horizontal intersections of real paths, which are not present in the real channel. The artifact paths increase the apparent diversity, but decrease the apparent capacity. The strength of any given artifact path depends on the number and strength of the real paths intersecting at that point. Because the overall power in the channel is kept constant through normalization, this has the effect of taking power away from real paths in the channel if they do not lie at the intersection of other paths. The end result is that the Kronecker model consistently underestimates capacity. Despite the evidence that suggests the Kronecker model to be inaccurate, it is by far the most popular MIMO channel model, mainly due to its simplicity and analytical tractability.

The effects of the separability assumption are best illustrated by way of an exemplar MIMO APS. Consider the case where we wish to approximate the spatial structure of a synthetic channel using the Kronecker model. Fig. 4 
illustrates the examplar MIMO APS, using an $8 \times 8$ system equipped with ULAs, whose antenna elements are separated by $\delta=\lambda / 2$. The channel consists of four equally-weighted correlated scatterers located at $\left(\phi_{\mathrm{Rx}}, \phi_{\mathrm{Tx}}\right)=\left(60^{\circ}, 40^{\circ}\right),\left(0^{\circ},-50^{\circ}\right),\left(-50^{\circ}, 60^{\circ}\right),\left(0^{\circ}, 0^{\circ}\right)$

In order to test the Kronecker model, we generate an ensemble of H-matrices using (13). We then compute the onesided correlation matrices $\mathbf{R}_{\mathrm{Rx}}$, and $\mathbf{R}_{\mathrm{Tx}}$ using (14). Finally, we compute the full correlation matrix $\mathbf{R}_{\mathrm{H}, \mathrm{Kron}}$ using (16), and use it to compute the MIMO APS. The Kronecker APS is plotted in Fig. 4(b). The Kronecker APS includes the four real paths, labeled A-D, and 8 artifact paths, labeled 1-8. Each artifact path occurs at the intersection of real paths. Artifact paths 4 and 5 are particularly strong, since they are both products of three real paths. Note also that the magnitudes of real paths $\mathrm{C}$ and $\mathrm{D}$ are greatly diminished as they do not lie at the intersection points of any other real paths. By extension, increasing the number of resolved scatterers will increase the number of artifact paths, and tends to diminish energy in paths which depart/arrive farther from the array boresight. This means that the Kronecker model performance degrades as we increase spatial resolution. We can increase spatial resolution, for example, by increasing the number of antennas and/or antenna directivity.

This analysis begs the question "how often does this happen in real channels?” We discuss this further in Section VIII.D using MIMO APSs computed from real data, and compare the results to those of the Kronecker and structured models.

\section{EXPERIMENTAL SETUP}

In the following, we briefly describe the specifications and experimental setup for both the WMSDR and the BYU wideband MIMO channel sounder. We use data gathered with both platforms to verify the structured channel model. We used the WMSDR to gather data in a fixed point-to-point outdoor environment. Measurements were taken at 15 locations. The BYU data were recorded using a much higher bandwidth than the WMSDR. The BYU data were gathered over eight fixed point-to-point locations indoors.

\section{A. WMSDR Specifications and Experimental Setup}

The WMSDR is a PC-based, highly versatile $4 \times 4$ wideband MIMO software defined radio. It consists of a separately mobile transmitter and receiver set.

\section{1) Transmitter Set}

We refer to all equipment at the transmit side collectively as the transmitter set. We present a block diagram of the transmitter set in Fig. 5. It consists of the WMSDR Transmitter, the transmitter PC, and external RF power amplifiers 
and antennas. Fig. 6 shows a picture of the complete transmitter set.

We generate an arbitrary complex baseband signal at the transmitter PC. The signal is fed directly to the WMSDR Transmitter. The WMSDR Transmitter houses four independent transmit chains which broadcast simultaneously. We can control each chain individually. The WMSDR Transmitter is also equipped with a Global Positioning System (GPS) time/frequency/position reference. This synchronizes the Local Oscillator (LO) and sample timing clocks to their counterparts in the WMSDR Receiver so that we can recover the sample time and phase during post processing. The entire transmitter set is housed in a mobile rack mount, along with RF power amplifiers, a battery back-up Uninterruptible Power Supply (UPS) and antenna array. These allow us to move the transmitter independent of the receiver outdoors, and without the use of a power outlet for a short period of time.

For the data considered below, we configured the WMSDR Transmitter to continuously transmit four independent maximal length sequences (m-sequences) of 4096 chips at 4Mchips/s using a BPSK constellation. We used a rectangular array of vertically polarized slot antennas with $70^{\circ} 3 \mathrm{~dB}$ beam width, and $4 \mathrm{dBi}$ gain at bore sight. Both transmitter and receiver work at a center frequency of $2135 \mathrm{MHz}$. The antennas are separated by $2 \lambda$ vertically, and $1.3 \lambda$ horizontally, where $\lambda$ is the wavelength at the center frequency. Two power amplifiers generate approximately 16dBm in-band power per antenna.

\section{2) Receiver Set}

We refer to all equipment at the receive side collectively as the receiver set. We present a block diagram of the receiver set in Fig. 7. The receiver set consists of the WMSDR Receiver, the receiver PC, and external Low Noise Amplifiers (LNAs) and antennas. The WMSDR Receiver houses four separate RF chains, agile LO, GPS time/frequency reference, and a microcontroller board to monitor the Automatic Gain Control (AGC) at the receiver front-end. The AGC allows a much greater dynamic range, which, in turn, allows for a greater variance in the received signal strength. Because the measurements were done in a static point-to-point environment, the AGC adjusted to one gain for each location and remained fixed for the duration of the measurement at that location. The WMSDR Receiver converts all four RF chains to digital complex baseband information simultaneously. The complex baseband information is recorded directly to disk at the receiver PC. As with the transmitter, all the above equipment is installed in a mobile rack mount. A picture of the receiver set is shown in Fig. 8.

For the data considered below, the receiver was equipped with a linear monopole array with $1.7 \lambda$ separation. To compute the MIMO PDP, we use a cross-correlation algorithm on the recorded data to find the beginning of each m- 
sequence. We then recover the sample timing and phase information. Once this is complete, we use matched filtering [15], extended to the MIMO case, to obtain the calibrated PDPs.

\section{3) Measurement Description}

For the measurements in Section VIII, we recorded samples at 8MSamples/s, for approximately 8ms continuous recording time per snapshot. At each receiver location, 5 snapshots were taken with about 100ms between snapshots. This means that, for each location, we recorded $35 \mathrm{H}$-matrices over approximately 440ms. The distance between the transmitter and receiver ranged from approximately $10 \mathrm{~m}$ to $100 \mathrm{~m}$. A grand total of $525 \mathrm{H}$-matrices were recorded over 15 locations. All locations were in an open parking lot at the McMaster University campus, with trees, cars, and buildings comprising the majority of the scatterers within 300m.

The test environment and equipment setup here were chosen specifically to test one extreme of the wideband MIMO channel. The relatively short propagation distance in a sparse scattering environment (a parking lot) combined with a directive antenna array at the transmitter, meant that the measured channel contained relatively few scatterers. In addition, the AGC at the receiver forced it to focus on the Line-of-Sight (LoS) path, and the contributions of most scatterers fell below the noise floor. This in turn meant that the measured channel offered relatively low diversity. Using data collected from this environment tests the structured models ability to approximate the spatial structure of low-diversity channels.

\section{B. BYU Wideband Channel Sounder Specifications and Experimental Setup}

The wideband MIMO channel sounder at BYU is based on an 8x8 switched antenna architecture, and is described in more detail in [16]. The following briefly describes the theory of operation and setup for BYU channel sounder for the data considered in Section VIII.

\section{1) Transmitter Set}

The BYU wideband transmitter functional diagram is shown in Fig. 9. The transmitter consists of a vector signal generator, rubidium clock source, an electronic 1:8 RF switch, a custom timing and synchronization (SYNC) unit, and associated antennas and amplifiers.

The vector signal generator was setup to generate 80 discrete tones at $1 \mathrm{MHz}$ spacing, for a total sounder bandwidth of $80 \mathrm{MHz}$. The output of the signal generator is connected to an electronic 1:8 RF switch, which distributes the RF signal to one of the eight antennas. The transmitter was set to generate 23dBm/antenna.

The RF switch position is determined by the SYNC unit. There is an identical SYNC unit at both transmitter and receiver, which are, in turn, synchronized to a rubidium reference. Before each data run, both SYNC units are 
synchronized to each other to minimize timing drift over the course of the measurement. Both RF sources are locked to a rubidium source, which minimizes relative frequency drift. The SYNC unit at the transmitter was programmed to excite each antenna for $50 \mu$ s. This means that, to scan the entire transmit array, we needed about $400 \mu$ s. The SYNC unit at the transmitter also ensures that the signal source sequence starts at the proper time instant.

2) Receiver Set

The receiver functional diagram is shown in Fig. 10. It consists of an electronic 8:1 RF switch, a PC equipped with a data acquisition card, SYNC unit, a rubidium clock source, and all associated RF hardware including amplifiers, antennas, Local Oscillator (LO), RF mixer, and bandpass filter.

The output of the RF switch is amplified by $40 \mathrm{~dB}$, mixed down to an Intermediate Frequency (IF), and fed to one channel of the data acquisition card (DAQ). The DAQ samples the signal at 500MSamples/s. The $10 \mathrm{MHz}$ signal from the Rubidium source is also sampled to enable phase coherence with the transmitter during post processing. Each receive antenna is sampled for $400 \mu \mathrm{s}$, the full cycle time of the transmitter. This means that it takes $3.2 \mathrm{~ms}$ to measure a complete MIMO channel response. Because of hardware limitations, there is a gap between each complete channel response measurement. For the data below, the receiver was programmed to measure a complete response every 25.6ms, and recorded data for up to $15 \mathrm{~s}$.

\section{3) Measurement Description}

The channel data were recorded at a center frequency of $2.55 \mathrm{GHz}$ and $5.2 \mathrm{GHz}$. Both transmitter and receiver employed a circular array of vertically polarized monopoles set to approximately $\lambda / 2$ spacing for each center frequency. Both the transmitter and receiver were located inside a building in separate rooms. The transmitter was fixed to one of two positions, and the receiver was moved to one of eight different locations. All measurements were done in a fixed point-to-point environment with no line of sight.

Compared with the WMSDR measurement setup described in Section VII.A, the BYU data was collected in a much more scatter-rich environment. Omni-directional antennas at both link ends ensure scatterers in the entire azimuth plane are equally well illuminated. The indoor environment provides numerous scatterers in the form of walls, floors, furniture, etc. In addition, the NLoS environment ensures that the receiver focuses on weaker reflected paths, which in turn increases diversity. The diversity present in the channel can be seen in the MIMO APSs presented in Section VIII.D, which shows many peaks over all AoA and AoD azimuths. The increased bandwidth versus the WMSDR also means that we have much better scatterer resolution. For example, for the WMSDR, the 
$2 \mathrm{MHz}$ effective bandwidth means that all energy within 500ns is grouped into one delay bin. Referring to Fig. 3, this means that each annulus is approximately $150 \mathrm{~m}$ bigger than the last. On the other hand, the BYU sounder's $80 \mathrm{MHz}$ bandwidth translates to an approximate scatter resolution of $3.75 \mathrm{~m}$. This data set was chosen specifically to test the structured model's ability to model scatter-rich environments.

\section{EXPERIMENTAL RESULTS}

\section{A. Methodology}

Using the PDPs computed from all data sets, we compute $\mathbf{R}_{\mathrm{wB}, \mathrm{H}}$, and use (28) to generate a synthetic H-tensor. We consider this to be our basis for comparison. Using the measured PDPs, we compute the one-sided correlation matrices $\mathbf{R}_{\mathrm{Rx}}, \mathbf{R}_{\mathrm{Tx}}$, and $\mathbf{R}_{\mathrm{D}}$. We then use eigendecomposition to compute the eigenbases $\mathbf{U}_{\mathrm{Rx}}, \mathbf{U}_{\mathrm{Tx}}$, and $\mathbf{U}_{\mathrm{D}}$. Using (32) we compute the wideband coupling coefficients $\omega_{\mathrm{ijk}}$ for $\mathrm{i}=1, \ldots, \mathrm{M}_{\mathrm{Rx}}, \mathrm{j}=1, \ldots, \mathrm{M}_{\mathrm{Tx}}$, and $\mathrm{k}=1, \ldots, \mathrm{D}$. We then use (33) to generate an ensemble of structured H-tensors $\mathcal{H}_{\text {struct }}$.

For comparison, we also generate an ensemble of H-tensors using the extended Kronecker model [8]. First, using the measured PDPs, we compute the one-sided correlation matrices $\mathbf{R}_{\mathrm{Rx}, \mathrm{d}}$, and $\mathbf{R}_{\mathrm{T} x \mathrm{~d}}$ at each delay tap. We use the Kronecker model to compute the narrowband H-matrix for each delay bin.

The capacity was computed using the formula [17]

$$
\mathrm{C}_{\mathrm{x}}=\frac{1}{\mathrm{~W}} \sum_{\mathrm{f}=1}^{\mathrm{F}} \log _{2}\left[\operatorname{det}\left(\mathbf{I}_{\mathrm{M}_{\mathrm{Rx}}}+\frac{\rho}{\mathrm{M}_{\mathrm{Tx}}} \tilde{\mathbf{H}}_{\mathrm{x}}[\mathrm{f}] \tilde{\mathbf{H}}_{\mathrm{x}}{ }^{\mathrm{H}}[\mathrm{f}]\right)\right] \Delta \mathrm{f}
$$

where $\mathrm{C}_{\mathrm{x}}$ is in bps/Hz, $\mathrm{W}=2$ (80) $\mathrm{MHz}$ with WMSDR (BYU) data, F is the number of frequency bins, $\rho$ is the system SNR, chosen to be $20 \mathrm{~dB}$, and $\Delta \mathrm{f}=\mathrm{W} / \mathrm{F}$. The system SNR is chosen such that the measurement SNR is guaranteed to be greater than the system SNR for all measurements. After converting $\mathcal{H}_{\text {synth }}$, $\mathcal{H}_{\text {kron }}$, and $\mathcal{H}_{\text {struct }}$ to the frequency domain, we map the result to $\tilde{\mathbf{H}}_{\mathrm{x}}[\mathrm{f}]$, where $\mathrm{X} \in\{$ synth, kron, struct $\}$. We also normalize $\tilde{\mathbf{H}}_{\mathrm{x}}[\mathrm{f}]$ using the normalization constant

$$
\mathrm{n}_{\mathrm{x}}=\sqrt{\frac{1}{\mathrm{M}_{\mathrm{Rx}} \mathrm{M}_{\mathrm{Tx}} \mathrm{F}} \sum_{\mathrm{i}=1}^{\mathrm{M}_{\mathrm{Rs}}} \sum_{\mathrm{j}=1}^{\mathrm{M}_{\mathrm{rz}}} \sum_{\mathrm{k}=1}^{\mathrm{F}}\left|\tilde{\mathrm{h}}_{\mathrm{x}, \mathrm{jk}}\right|^{2}}
$$

where $\tilde{h}_{x, j \mathrm{jk}}$ is the $\mathrm{ijk} \mathrm{k}^{\text {th }}$ element of $\tilde{\mathbf{H}}_{\mathrm{x}}[\mathrm{f}]$. 


\section{B. Results: WMSDR Data Set}

Table 1 summarizes the capacity results for the case when $\left(M_{R x}, M_{T x}, D\right)=(4,4,4)$ averaging over an ensemble of 10,000 iterations. The number of parameters needed to compute $\mathcal{H}_{\text {synth }}, \mathcal{H}_{\text {Kron }}$ and $\mathcal{H}_{\text {struct }}$ are listed in Table 2. Fig. 11 shows the modeled versus measured capacity for the WMSDR data, using the structured model and the extended Kronecker model. The diagonal represents the case of no model error. Model error is defined as

$$
\% \text { Error }=\frac{C_{x}-C_{\text {synth }}}{C_{\text {synth }}} \times 100
$$

where $\mathrm{X} \in\{$ Kron, struct $\}$.

In general, the structured model performed very well, especially when compared to the extended Kronecker model. For all locations, the average structured model error is $4.4 \%$, while the average model error for the extended Kronecker model is 33.9\%. In Fig. 11 we see the Kronecker model consistently underestimates the capacity of the channel. These results are in line with those given by Yu et al. [8].

\section{Results: BYU Data Set}

The BYU data are divided into two larger data sets, one recorded at 2.55GHz (Data 255), and the second at $5.2 \mathrm{GHz}$ (Data 52). Each set contains data gathered at 8 different locations. Tables 3 and 4 list the results for the average capacity error over all locations for Data 255 and Data 52 respectively. For each data set, we consider the cases when $\mathrm{M}_{\mathrm{Rx}}=\mathrm{M}_{\mathrm{Tx}}=4,6$, and 8 , and $\mathrm{D}=4,7$, and 10. Fig. 12 shows modeled versus measured capacity graph for all locations, all data sets, and all cases listed above. Fig. 13 shows the Cumulative Distribution Function (CDF) for all locations, all data sets, and all cases. As mentioned in Section IV.A, increasing $M_{R x}, M_{T x}$ increases the average Kronecker model error. The structured model error however remains relatively constant as we increase the array size.

Table 5 shows the average model error broken down for the cases when $M_{R x}=M_{T x}=4,6$, and 8. As with the WMSDR data, our model performed quite well overall. The total average error, averaging over all data sets and all cases mentioned above, was $46.1 \%$ for the extended Kronecker model, and $4.1 \%$ for the structured model. The structured model error for the BYU data is close to that of the WMSDR data, which was $4.4 \%$.

\section{Results: $M I M O A P S$}

Using $\mathcal{H}, \mathcal{H}_{\text {Kron }}$ and $\mathcal{H}_{\text {struct }}$ computed from the BYU data, we compute the correlation matrices $\mathbf{R}_{\mathrm{WB}, \mathrm{H}[\mathrm{d}] \mathrm{H}[\mathrm{d}]}$, 
$\mathbf{R}_{\mathrm{WB}, \mathrm{H}[\mathrm{d}] \mathrm{H}[\mathrm{d}], \mathrm{Kron}}$, and $\mathbf{R}_{\mathrm{WB}, \mathrm{H}[\mathrm{d}] \mathrm{H}[\mathrm{d}] \text {,struct }}$ for each delay d. We then compute the MIMO APS for each correlation matrix at each delay. This provides a qualitative indication of how well the Kronecker and structured models represent the true spatial structure of the channel. Fig. 14 illustrates the MIMO APS for all three correlation matrices at location 1, set 1b, for the first delay. The true MIMO APS, shown in Fig. 14(a), contains many distinct resolvable paths, indicating a scatter-rich environment. Following the discussion in Section VI, the Kronecker APS, shown in Fig. 14(b) emphasizes the paths closest to the AoA $=\mathrm{AoD}=0^{\circ}$ axes, while de-emphasizing all other paths. This creates a noticeable cross pattern. The structured model, used to produce the APS shown in Fig. 14(c), does a much better job of representing the center of most major paths, regardless of their azimuth location.

In general, for most locations, the structured model APS followed much more closely to the true MIMO APS than did the Kronecker APS. The Kronecker model faired worse in channels where strong scatterers were located away from the AoA-AoD axes, or had many diagonally-aligned peaks. With respect to the BYU data, this was usually the case. Thus, for real environments, the Kronecker model does not represent the spatial structure as well as the structured model.

\section{CONCLUSION}

This paper presented a new wideband MIMO channel model, which we termed the structured model. We first introduced the concept of an H-tensor, which characterizes the channel gain of a wideband MIMO channel. We then defined the wideband correlation matrix. We extended the concept of one-sided correlation to three dimensions. We then presented the structured model, which generates an ensemble of H-tensors using the measured one-sided correlation matrices. A distinct advantage of the structured model is that it doesn't assume independence between scatterers at the transmitter and receiver, and it also models the correlation between scatterers at different delays. We presented specifications for a new MIMO SDR, dubbed the WMSDR. Using data from the WMSDR, and BYU's wideband channel sounder, we showed that our model performed very well with respect to predicting the capacity of the channel. Both data sets used were gathered in two very different environments and using different system bandwidths. This proved the robustness of the structured model. We also showed that the structured model performs much better than the extended Kronecker model, despite using fewer parameters to generate an ensemble of $\mathrm{H}$ tensors. Finally, we showed, using MIMO APSs, that the structured model captures the spatial structure of the channel better than the Kronecker model. 


\section{ACKNOWLEDGMENT}

The authors wish to express our deepest gratitude to Mike Jensen at Brigham Young University, USA for access to BYU data and many fruitful discussions. The authors are also deeply indebted to Tricia Willink at the Communications Research Centre, Canada for useful discussions that led to many of the ideas in this paper. The authors also owe many thanks to Tim Davidson of McMaster University, Canada for much needed help while working on the theory behind the structured model, for many discussions, and for feedback on this paper. The authors wish to thank Mathini Sellathurai at Cardiff University, UK for references and feedback on this paper. Finally, the authors would also like to thank David Lindo and Debby Mavriyannakis for all their help collecting the WMSDR data. 
TABLES

TABLE 1: KRONECKER VERSUS STRUCTURED MODEL ERROR

\begin{tabular}{|c|c|c|c|c|c|}
\hline Loc. & $\begin{array}{l}\mathrm{C}_{\text {synth }} \\
\text { (bps/ } \\
\mathrm{Hz} \text { ) }\end{array}$ & $\begin{array}{l}\mathrm{C}_{\text {kron }} \\
\text { (bps/ } \\
\mathrm{Hz} \text { ) }\end{array}$ & $\begin{array}{l}\mathrm{C}_{\text {struct }} \\
\text { (bps/ } \\
\mathrm{Hz} \text { ) }\end{array}$ & $\begin{array}{l}\% \\
\text { Error } \\
\text { Kron }\end{array}$ & $\begin{array}{l}\% \\
\text { Error } \\
\text { Struct }\end{array}$ \\
\hline 0901 & 16.7 & 9.6 & 16.3 & 42.5 & 2.4 \\
\hline 1100 & 15.0 & 10.4 & 15.3 & 30.7 & 2.0 \\
\hline 1215 & 17.7 & 11.3 & 17.4 & 36.2 & 1.7 \\
\hline 1354 & 17.1 & 8.7 & 16.9 & 49.1 & 1.2 \\
\hline 1750 & 17.1 & 12.2 & 16.5 & 28.7 & 3.5 \\
\hline 1844 & 19.7 & 11.7 & 18.7 & 40.6 & 5.1 \\
\hline 1931 & 17.0 & 10.9 & 16.0 & 35.9 & 5.9 \\
\hline 2021 & 16.6 & 10.7 & 15.9 & 35.5 & 4.2 \\
\hline 2116 & 16.6 & 11.1 & 16.6 & 33.1 & 0 \\
\hline 2537 & 14.9 & 10.8 & 15.3 & 27.5 & 2.7 \\
\hline 2652 & 11.2 & 8.8 & 12.4 & 21.4 & 10.7 \\
\hline 2744 & 12.6 & 9.8 & 14.0 & 22.2 & 11.1 \\
\hline 2841 & 15.8 & 11.6 & 15.0 & 26.6 & 5.1 \\
\hline 3108 & 13.7 & 8.7 & 14.9 & 36.5 & 8.8 \\
\hline 3223 & 15.7 & 9.0 & 15.9 & 42.7 & 1.3 \\
\hline \multicolumn{4}{|r|}{ Error: } & 33.9 & 4.4 \\
\hline
\end{tabular}

TABLE 2: PARAMETER COMPARISON

\begin{tabular}{lll}
\hline Model & Number Param. $\left(\mathrm{M}_{\mathrm{Rx}}, \mathrm{M}_{\mathrm{Tx}}, \mathrm{D}\right)$ & $\begin{array}{l}\text { Number } \\
\text { Param. } \\
(4,4,4)\end{array}$ \\
\hline $\mathcal{H}_{\text {synth }}$ & $\left(\mathrm{M}_{\mathrm{Rx}} \mathrm{M}_{\mathrm{Tx}} \mathrm{D}\right)^{2}$ & 4096 \\
$\mathcal{H}_{\text {kron }}$ & $\mathrm{D}\left(\mathrm{M}_{\mathrm{Rx}}^{2}+\mathrm{M}_{\mathrm{Tx}}^{2}\right)$ & 128 \\
$\mathcal{H}_{\text {struct }}$ & $\left(\mathrm{M}_{\mathrm{Rx}} \mathrm{M}_{\mathrm{Tx}} \mathrm{D}\right)+\left(\mathrm{M}_{\mathrm{Rx}}^{2}+\mathrm{M}_{\mathrm{Tx}}^{2}+\mathrm{D}^{2}\right)$ & $\mathbf{1 1 2}$ \\
\hline \hline
\end{tabular}

TABLE 3: MODEL ERROR FOR DATA 255

\begin{tabular}{|c|c|c|}
\hline$M_{R x}, M_{T x}, D$ & $\begin{array}{l}\text { \% Error } \\
\text { Kronecker }\end{array}$ & $\begin{array}{l}\% \text { Error } \\
\text { Structured }\end{array}$ \\
\hline $4,4,4$ & 42.6 & 4.8 \\
\hline $4,4,7$ & 37.1 & 4.5 \\
\hline $4,4,10$ & 35.5 & 4.6 \\
\hline $6,6,4$ & 52.3 & 4.6 \\
\hline $6,6,7$ & 48.6 & 3.4 \\
\hline $6,6,10$ & 46.9 & 3.2 \\
\hline $8,8,4$ & 60.1 & 6.1 \\
\hline $8,8,7$ & 55.1 & 4.3 \\
\hline $8,8,10$ & 53.8 & 4.4 \\
\hline Average: & 47.7 & 4.4 \\
\hline
\end{tabular}


TABLE 4: MODEL ERROR FOR DATA 52

\begin{tabular}{lll}
\hline \hline \multirow{2}{*}{$\mathrm{M}_{\mathrm{Rx}}, \mathrm{M}_{\mathrm{Tx}}, \mathrm{D}$} & $\begin{array}{l}\text { \% Error } \\
\text { Kronecker }\end{array}$ & $\begin{array}{l}\text { \% Error } \\
\text { Structured }\end{array}$ \\
\hline $4,4,4$ & 39.8 & 3.8 \\
$4,4,7$ & 35.5 & 4.5 \\
$4,4,10$ & 34.7 & 3.9 \\
$6,6,4$ & 51.1 & 4.7 \\
$6,6,7$ & 47.1 & 3.5 \\
$6,6,10$ & 45.1 & 2.9 \\
$8,8,4$ & 58.2 & 5.2 \\
$8,8,7$ & 54.2 & 3.3 \\
$8,8,10$ & 53.0 & 3.2 \\
\hline Average: & 44.4 & $\mathbf{3 . 7}$ \\
\hline \multicolumn{4}{c}{ TABLE 5: AvERAGE MODEL ERROR BY ARRAY sIzE } \\
\hline \hline M & $\%$ Error & $\%$ Error \\
Rx & Kronecker & Structured \\
\hline 4,4 & 34.6 & 4.4 \\
6,6 & 48.3 & 3.6 \\
8,8 & 55.4 & 4.3 \\
\hline Total Average: & 46.1 & $\mathbf{4 . 1}$ \\
\hline \hline
\end{tabular}




\section{FIGURES}

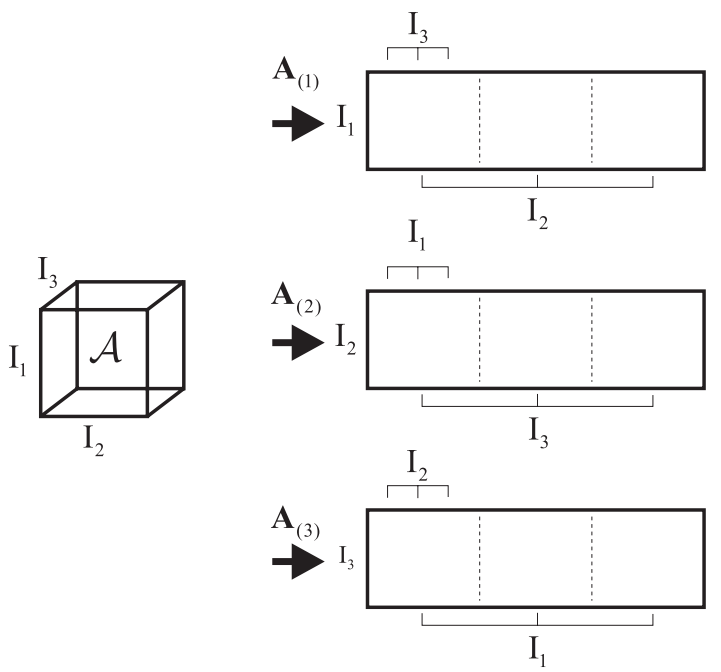

Fig. 1. Illustrating the three different matrix unfoldings for a third-order tensor $\mathcal{A}$.

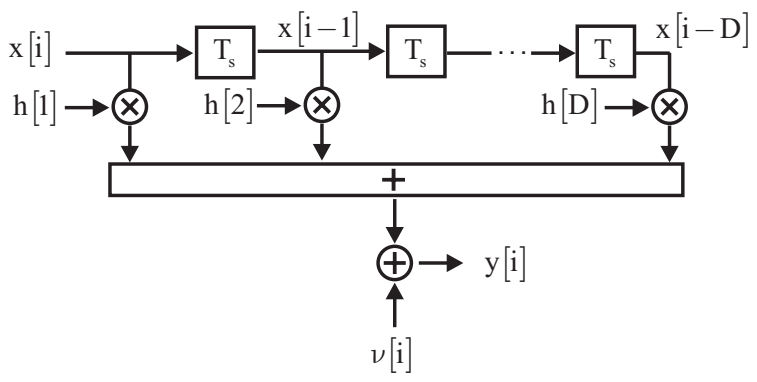

Fig. 2. The symbol-spaced discrete time baseband equivalent tapped-delay line model.

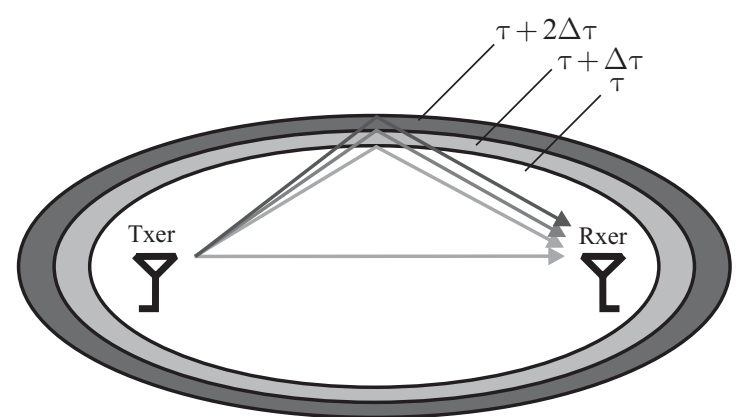

Fig. 3. Structure of the wideband SISO channel showing the oval of scatterers topology where $\tau=1 / \mathrm{W}$ for system bandwidth W. 


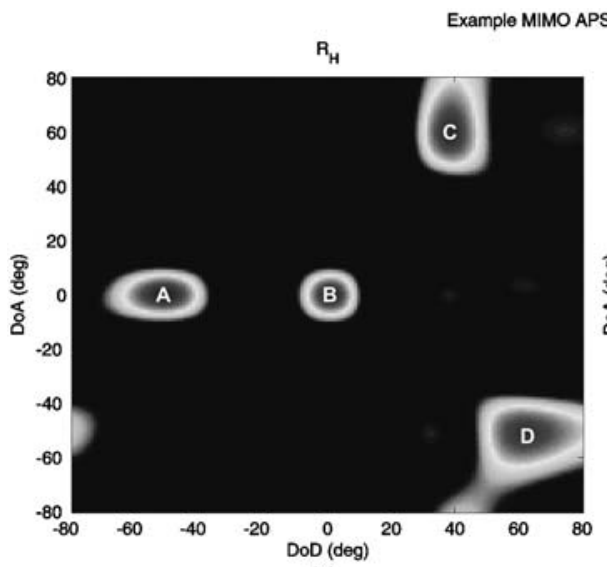

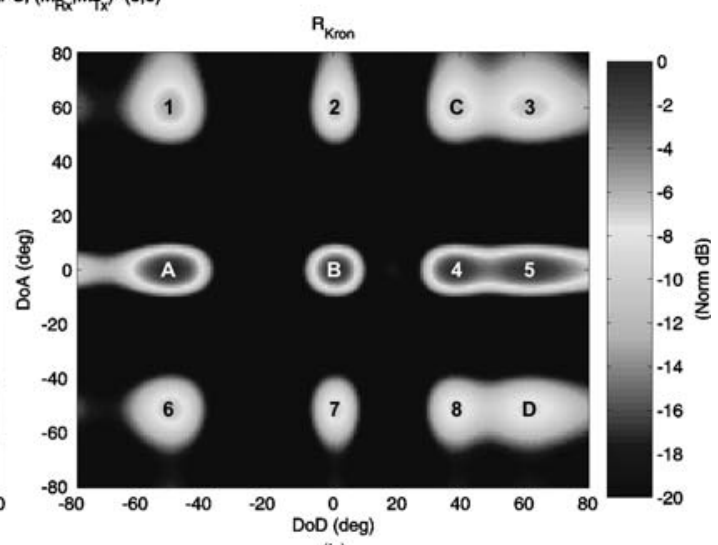

Fig. 4. Example MIMO APSs. (a) Example using full correlation matrix, 4 distinct scatterers. (b) Kronecker model approximation showing additional artifact paths.

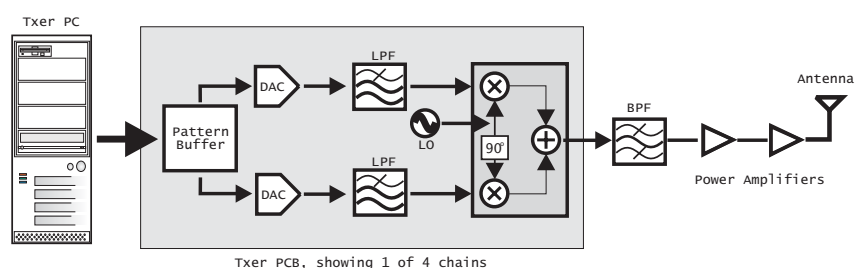

Fig. 5. The WMSDR transmitter set block diagram showing 1 of 4 chains.

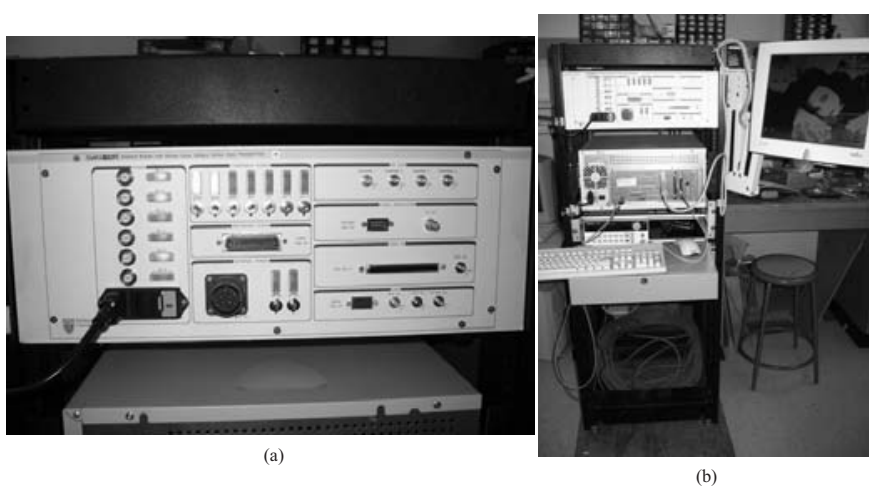

Fig. 6. The WMSDR Transmitter (a), and the transmitter set (b).

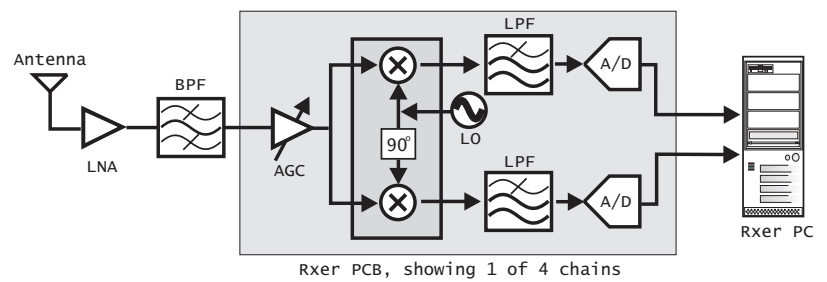

Fig. 7. The WMSDR receiver set block diagram. 


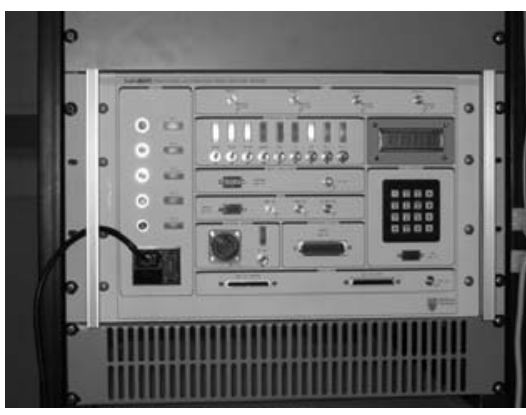

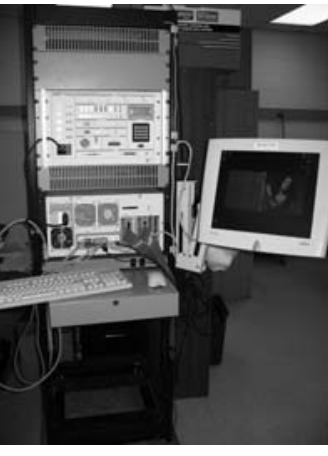

(b)

Fig. 8. The WMSDR Receiver (a), and the receiver set (b).

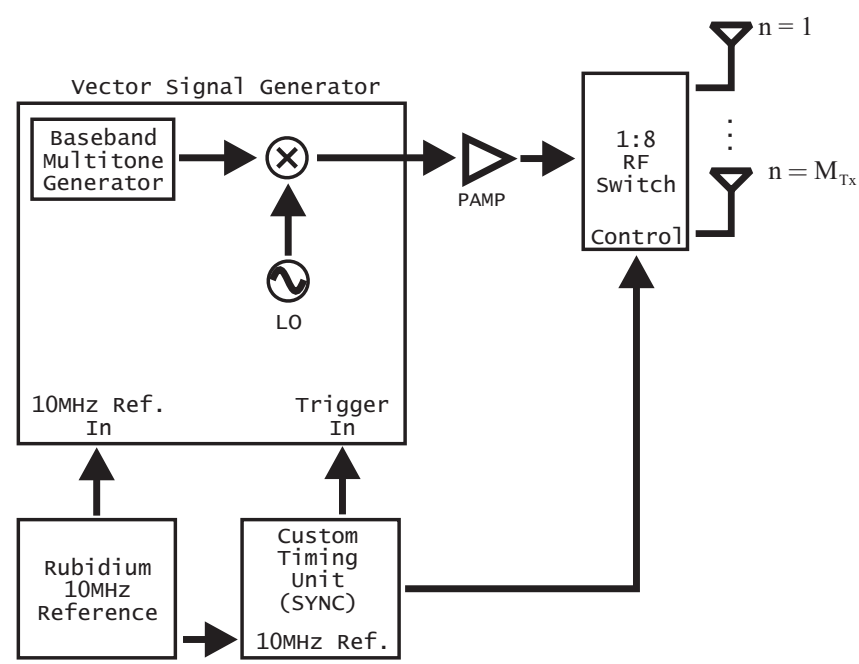

Fig. 9. The BYU wideband transmitter block diagram.

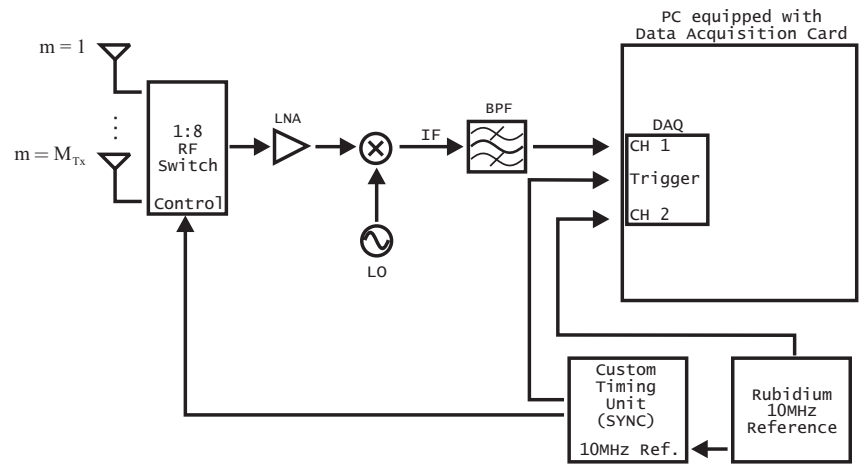

Fig. 10. The BYU wideband receiver block diagram. 


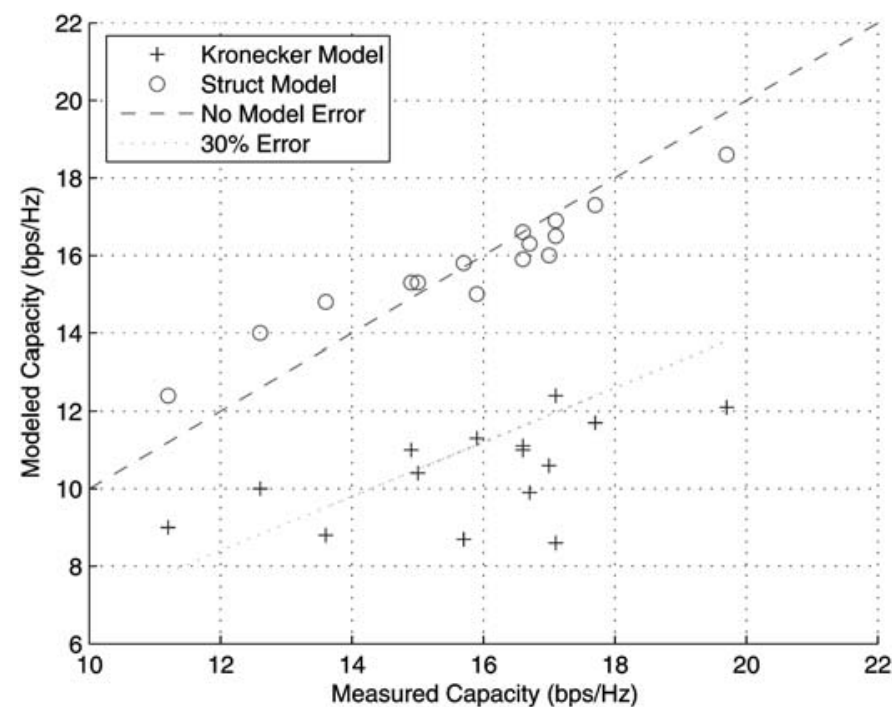

Fig. 11. Modeled versus measured capacity for both Kronecker and Structured models using the WMSDR data. The diagonal line represents no model error.

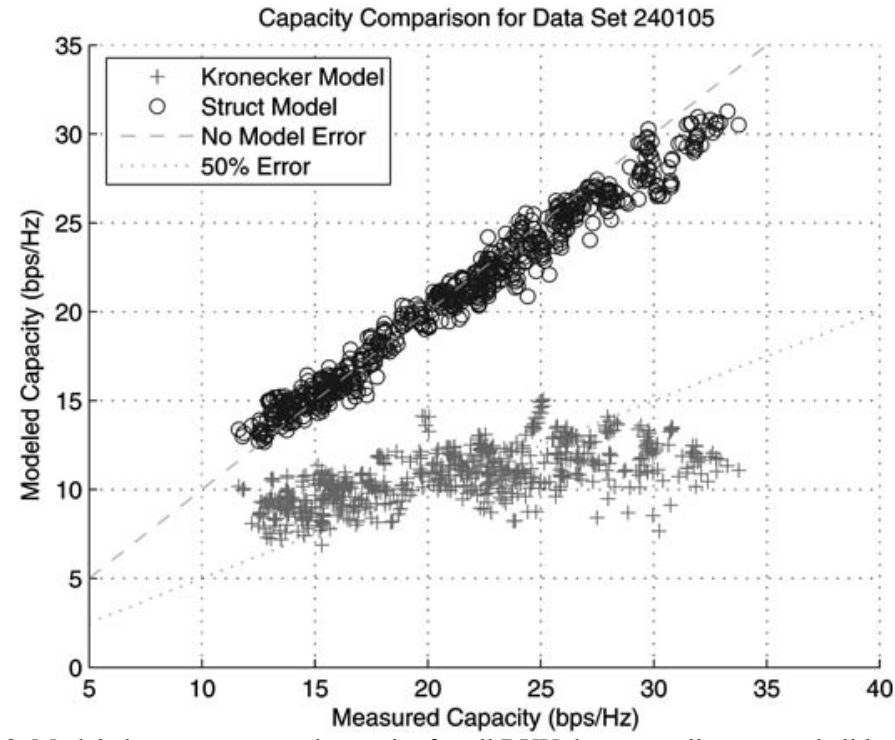

Fig. 12. Modeled versus measured capacity for all BYU data sets, all cases, and all locations.

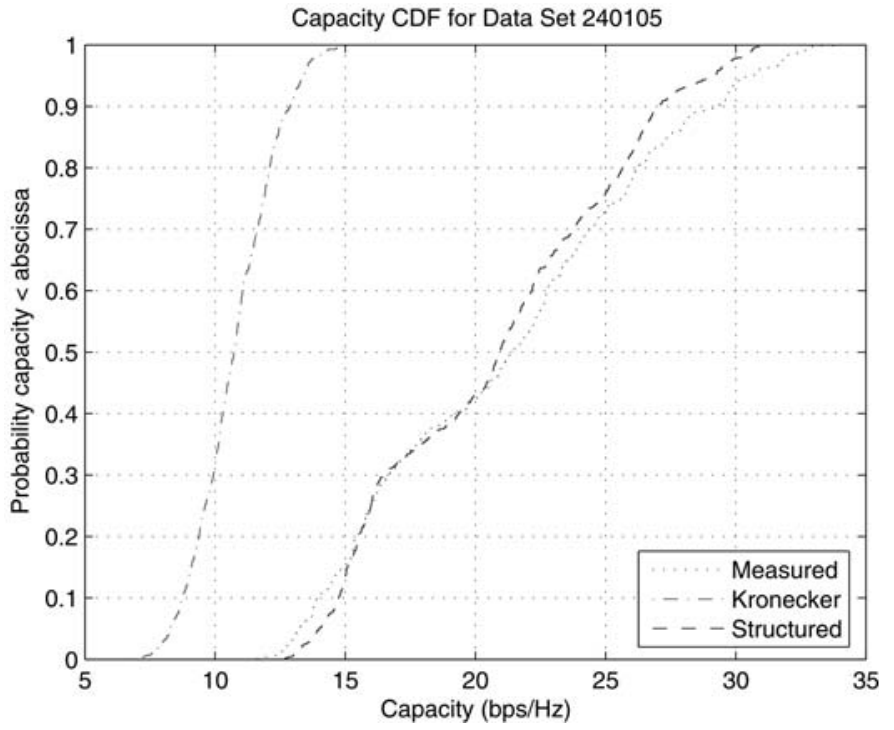

Fig. 13. Capacity CDF for all data sets, all cases, and all locations. 


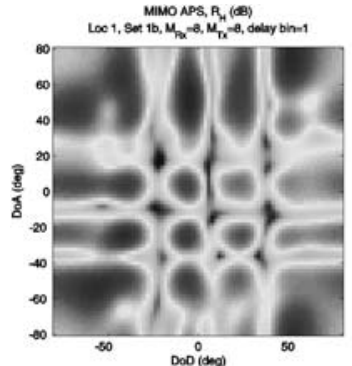

(a)
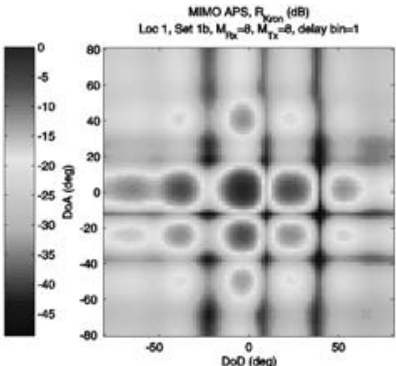

(b)

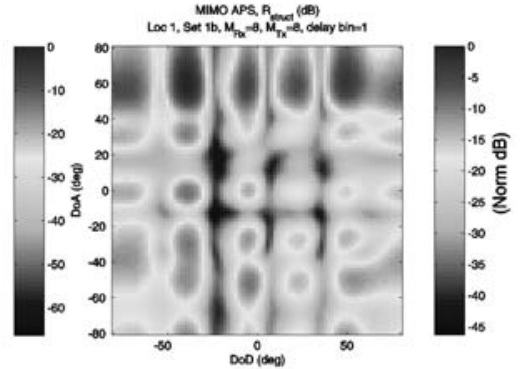

(c)

Fig. 14. MIMO APSs computed using BYU data, showing (a) APS from $\mathbf{R}_{\mathrm{H}}$, (b) APS from $\mathbf{R}_{\text {Kron }}$, and (c) APS from $\mathbf{R}_{\text {struct }}$

\section{REFERENCES}

[1] E. Telatar, "Capacity of multi-antenna Gaussian channels,” European Transactions on Telecommunications, Vol. 10, No. 6, pp. 585-595, Nov-Dec 1999.

[2] G.J. Foschini, M.J. Gans, “On limits of wireless communication in a fading environment when using multiple antennas,” Wireless Personal Commun., vol. 6, no. 3, pp. 311-335, March. 1998.

[3] L. Zheng, D.N.C. Tse, “Diversity and multiplexing; a fundamental tradeoff in multiple antenna channels,” IEEE Trans. Information Theory, vol. 49, no. 5, pp. 1073-1096, May 2003.

[4] D.-S. Shiu, G.J. Foschini, M.J. Gans, and J.M. Kahn, "Fading correlation and its effect on the capacity of multielement antenna systems," IEEE Trans. Commun., vol. 48, pp. 502-513, Mar 2000.

[5] D. Chizhik, J. Ling, P.W. Wolniansky, R.A. Valenzuela, N. Costa, and K. Huber, "Multiple-intput-multiple-output measurements and modeling in Manhattan,” IEEE J. on Selected Areas in Commun., vol. 21, pp. 321-331, April 2003.

[6] J.W. Wallace, M.A. Jensen, A.L. Swindlehurst, B.D. Jeffs, "Experimental characterization of the MIMO wireless channel: data acquisition and analysis," IEEE Trans. Wireless Commun., vol. 2, no. 2, March 2003.

[7] W. Weichselberger, M. Herdin, H. Özcelik, E. Bonek, “A stochastic MIMO channel model with joint correlation of both link ends,” IEEE Trans. Wireless Commun., vol. 5, no. 1, January 2006.

[8] K. Yu, M. Bengtsson, B. Ottersten, D. McNamara, P. Karlsson, M. Beach, "Modeling of the wide-band MIMO radio channels based on NLoS indoor measurements,” IEEE Trans. Vehicular Tech., vol. 53, no. 3, May 2004.

[9] Werner Weichselberger, Spatial Structure of Multiple Antenna Radio Channels, Ph.D Dissertation, Technische Universtät Wien, Austria, December 2003.

[10] L. De Lathauwer, Signal Processing Based on Multilinear Algebra, Ph.D Thesis, Katholieke Universiteit Leuven, Belgium, September 1997.

[11] J.H. Heinbockel, Introduction to Tensor Calculus and Continuum Mechanics. Avalable: http://www.math.odu.edu/ jhh/part1.PDF, 1996.

[12] J.G. Proakis, Digital Communications, 3rd Ed., McGraw-Hill, San Francisco, 1995.

[13] E. Bonek, M. Herdin, W. Weichselberger, H. Özcelik, "MIMO-study propagation first!,” Proc. International Symposium on Signal Processing and Information Tech. 2003, 14-17 December 2003 pp. 150-153.

[14] H. Özcelik, M. Herdin, W. Weicheselberger, J. Wallace, E. Bonek, “Deficiencies of the Kronecker MIMO radio channel model,” IEE Electronics Letters, vol. 39, pp. 1209-1210, August 2003.

[15] P.C. Fannin, A. Molina, S.S. Swords, P.J. Cullen, “Digital signal processing techniques applied to mobile radio channel sounding,” IEE Proc.-F, vol. 138, no. 5, October 1991.

[16] B.T. Maharaj, L.P. Linde, J.W. Wallace, M.A. Jensen, "A cost-effective wideband MIMO channel sounder and initial co-located 2.4 GHz and 5.2 GHz measurements,” Proc. International Conf. on Acoustics, Speech, and Signal Processing 2005, Vol. 3, 18-23 March 2005 pp. 981-984.

[17] N. Skentos, A.G. Kanatas, G. Pantos, P. Constantinou, "Capacity results from short range fixed MIMO measurements at 5.2GHz in urban propagation environment,” Proc. International Conf. on Commun. 2004, vol. 5, June 2004, pp. 3020 - 3024. 